\title{
Modelo Gestión de Calidad centrado en la formación integral del Médico por Competencias
}

\author{
Haydeé Parra Acosta \\ Facultad de Medicina de la UACH \\ hparra@uach.mx \\ Julio César López González \\ Facultad de Medicina de la UACH \\ jclopez@uach.mx \\ Ma. Elena Martínez Tapia \\ Facultad de Medicina de la UACH \\ maelmata@prodigy.net.mx
}

\section{Resumen}

Los resultados de un estudio comparativo entre médicos internos de pregrado (MIP), egresados con el plan curricular tradicional (Flexneriano) y plan curricular por competencias, evidenciaron que existen limitaciones en el desempeño profesional de los Médicos de ambos planes curriculares; no presentaron diferencias significativas. También mostró que existen carencias en la administración educativa del currículo, respecto a la formación del médico en el internado de pregrado y las tutorías clínicas. Precedente para el diseño de un Modelo de Gestión de Calidad de la Facultad de Medicina de la UACH que contribuye al desempeño profesional por competencias del MIP. El método que se utilizó, investigación-acción a través de cinco fases: 1. Formación y actualización del comité de investigación y de los participantes en el diseño del modelo. 2. Identificación y análisis del problema. 3. Precisión de lo que se pretende mejorar en la formación médica durante el Internado de Pregrado. 4. Estructuración del Modelo de Gestión de Calidad. 5. Implementación y seguimiento del Modelo. Los resultados conforman un Modelo de Gestión de Calidad acorde: al programa educativo de la Facultad, los lineamientos del CIFRHS, los estándares propuestos por COMAEM, las políticas y normativas para el internado de pregrado; que contribuye al mejoramiento continuo del proceso formativo de los MIP por competencias a través de cuatro acciones: direccionar, planear, actuar y evaluar; correspondiente a un ciclo de mejoramiento continuo.

Palabras clave

Gestión de Calidad, Competencias, Administración Educativa, Evaluación,

Tutorías clínicas.

\section{Introducción}

Los problemas de salud en el contexto actual, demandan prestar la atención en la formación integral de los médicos por competencias. Ante ello, la Facultad de Medicina de la UACH, innovó su currículo en el 2006, mediante un 
proceso participativo con 41 docentes y 8 estudiantes y, lo evaluó en el 2009 a través del Comité de Evaluación Académica. Así mismo, al coincidir la primera generación formada con el plan de estudios basado en competencias y la última del plan de estudios tradicional (Flexner) utilizado por más de 50 años en el Internado de Pregrado en Junio de 2010; se consideró oportuno evaluar las dos generaciones a través de un estudio comparativo. Esta investigación integra tres Etapas: I. Diagnóstico, II. Diseño del Modelo de Gestión de Calidad e Implementación, que nos ocupa en este trabajo y, III. Seguimiento y Evaluación del Modelo de Gestión de Calidad.

En la Fase I, se aplicó un Examen Clínico Objetivo Estructurado (ECOE) a 56 médicos internos de pregrado (MIP), 28 de cada generación para evaluar la competencia clínica con pacientes estandarizados en 18 estaciones con: lista de cotejo y rúbrica. La información se procesó utilizando la prueba T de Student con un nivel de significancia de $\mathrm{p}$ $<0,05$. Se administró un cuestionario a 266 personas y 365 MIP para analizar la relación entre la administración educativa del plan de estudios basado en competencias y su desempeño. Los resultados del ECOE mostraron que no hay diferencias significativas en ambas generaciones. En la lista de cotejo, el 70\% de los MIP obtuvo valores promedio de $53.90 \pm 0.57$. Aprobaron 10 de 18 estaciones de acuerdo al pase de estándar, establecido por los médicos evaluadores.

Los resultados del cuestionario mostraron deficiencias en la administración educativa del currículo respecto a: tiempo asignado para análisis de casos, personal de supervisión, asesoría (Tutoría clínica) y evaluación $\mathrm{y}$, en el cumplimiento de los objetivos del internado de pregrado. Otras limitantes referidas son bibliohemeroteca y laboratorio de anatomía patológica. Observándose, que un buen plan de estudios es esencial para favorecer el desarrollo de las competencias propuestas en el perfil de egreso pero no suficiente, es importante propiciar las condiciones administrativas y académicas que contribuyan a ello. Ante ello se planteó la siguiente interrogante:

¿Qué aspectos administrativos y académicos favorecen una formación médica de calidad por competencias en el Internado de Pregrado?.

\section{Objetivo de investigación}

Diseñar un Modelo de Gestión de Calidad de la Facultad de Medicina que contribuya al mejoramiento continuo del desempeño profesional por competencias del médico interno de pregrado, diseñado de forma colaborativa entre: docentes, jefes de enseñanza, residentes y coordinador del internado de pregrado.

\section{Objetivos específicos}

1. Contribuir a la formación del comité de investigación y del personal que apoya la formación de los MIP sobre modelos de gestión en la formación médica.

2. Precisar las limitantes y necesidades que se presentan en el desempeño profesional de los médicos y en la administración educativa del currículo, con los MIP y el personal que apoya su formación para plantear estrategias que contribuyan a mejorar el proceso de formación médica en congruencia con el contexto social.

3. Diseñar de forma participativa el Modelo de Gestión de Calidad a través de proyectos que den respuesta a los problemas y necesidades identificados en el internado de pregrado

4. Conformar de forma participativa el manual de procedimientos para la implementación de los proyectos que integran el Modelo de Gestión. 


\section{Proceso metodológico}

El enfoque de este proceso investigativo es cualitativo. El diseño del Modelo de Gestión de Calidad se realizó mediante un proceso participativo a través de la investigaciónacción, un método que pone énfasis en la reflexión de un grupo de participantes para la acción en la acción, con el propósito de transformarla (Barabtarlo, 2005).

\section{Fases}

1. Formación y actualización del comité de investigación y de los participantes en el diseño del modelo.

Desarrollo de un Seminario Taller con apoyo de experto en la gestión para la formación en competencias (GESFOC).

2. Identificación y análisis del problema principal de cada uno de los elementos de la administración del currículo.

Análisis de los resultados de la Etapa I de la investigación. Identificación de las limitantes que se presentan en el desempeño profesional de los médicos y en la administración educativa del currículo.

3. Precisión de lo que se quiere mejorar o transformar para mejorar la formación médica en el internado de pregrado.

Equipos de trabajo con asistentes al Seminario taller de acuerdo a los elementos analizados: administración educativa del currículo, organización y estructura de los contenidos, formación y evaluación de competencias, tutorías clínicas e infraestructura y equipamiento. Aplicación de entrevista de grupo focal a médicos internos de pregrado.

4. Estructuración del Modelo de gestión de calidad

A partir de la identificación y análisis del problema, así como de los aspectos a mejorar, diseñar los proyectos que integrarán el Modelo de Gestión de Calidad, considerando las siguientes actividades.

Seleccionar los estándares de calidad propuestos por COMAEM que respondan a lo que se pretende mejorar.
Identificar los indicadores y criterios del acuerdo al estándar.

Diseñar rúbricas y/o mapas para valorar los criterios.

Establecer las actividades factibles y pertinentes que contribuyan al logro del cumplimiento del estándar.

Desarrollar el Gantt (gráfico que permite visualizar la planeación de las actividades, los recursos que se requieren así como los responsables).

\section{Implementación y seguimiento}

Diseño del manual de procedimientos que asegure la implementación del modelo de gestión acorde al programa educativo de la Facultad, los lineamientos correspondientes del CIFRHS, los estándares propuestos por COMAEM y a las políticas y normativas para el internado de pregrado.

\section{Participantes}

Coordinador del internado de pregrado, jefes de enseñanza, cordinadores de área, residentes, docentes, presidentes de academias y personal que integra el comité de investigación educativa.

\section{Procesamiento y análisis de la información} El análisis de los datos cualitativos se realizó tomando como base los aportes de Creswell (2003) y Rodríguez Gómez et al. (1996) en Guzmán (2009) con la siguiente secuencia de pasos:

1. Organización y preparación de la información recuperada en el seminariotaller.

2. Lectura de la información recuperada en su conjunto para comprender el sentido general y reflexionar sobre el significado global.

3. Análisis de la información e identificación de unidades de análisis; que corresponden lo más representativo que mencionaron los participantes durante el seminariotaller y en las reuniones de trabajo. 
1. Construcción de categorías de contenido.

4. Tomando como base los patrones derivados de la separación en unidades bajo el criterio temático; se agruparon categorías. Así mismo, se diseñó un gráfico.

5. Elaboración del informe del Modelo de gestión y de los proyectos generados. Este paso consistió en integrar las categorías construidas en el informe de resultados, tomando como referente el objetivo de investigación

\section{Resultados por Fases}

Fase 1. Formación y actualización

Se desarrolló un Seminario-Taller con apoyo del Dr. Sergio Tobón Tobón, donde se proporcionaron las bases teóricas y metodológicas correspondientes a la estructura del modelo de gestión de calidad para la formación de competencias $y$, se precisaron los elementos que lo conforman: administración educativa del currículo, organización y estructura de contenidos, evaluación de competencias, tutorías clínicas e infraestructura y equipamiento.

Este seminario contó con la presencia de 72 participantes.

\section{Fase 2. Identificación y análisis del problema}

En el seminario-taller, los integrantes del comité de investigación educativa, integraron equipos de trabajo con los asistentes de acuerdo a cada uno de los elementos del modelo de gestión para identificar la problemática que subyace en el Internado de pregrado.

El personal que apoya la formación de los médicos internos de pregrado, manifestó que el internado, representa una oportunidad en la formación médica; no obstante, expresan que los médicos internos, no desarrollan las competencias por falta de práctica, iniciativa y apatía. Y que la mayor parte del tiempo que pasan en el Internado lo dedica a cuestiones administrativas, al llenado de documentación. Así se muestra en los siguientes comentarios:

"Es una oportunidad para desarrollar habilidades medicas dentro de un hospital.

"Les hace falta iniciativa..."

"Les falta disponibilidad, responsabilidad, conocimientos $\mathrm{y}$ presentación"

"Considero que hay problemas de actitud, no tanto de conocimientos. .."

"Están dedicados solamente a la documentación y/o administrativo del paciente y no a los procedimientos o atención oportuna al paciente hospitalizado"

"El problema es que predominantemente realizan papelería y procedimientos mínimos y repetitivos, falta de horas de enseñanza"

Al ponderar las cuestiones administrativas sobre la práctica médica frente a situaciones clínicas de salud, se observa que la formación integral del estudiante no constituye la justificación del internado de pregrado. García, Tobón y López, 2009).

Expresaron también, la importancia de conocer los objetivos del internado de pregrado, mejorar la supervisión de los MIP por parte de la Facultad, organizar los servicios y rotaciones así como horarios de guardias y clases.

"Hay que saber cuáles son los objetivos del internado"

"De acuerdo a la estructura hospitalaria considero deberían ser supervisados por la Facultad de Medicina y no dejar todo a cargo del Médico Residente o adscrito del servicio y así cumplirían más los objetivos"

"Que la persona encargada de su supervisión pase más tiempo con ellos" 
"Es importante mayor supervisión de las historias clínicas si cumple con las rotaciones básicas de hospitalizaciones pero también deben participar en las consultas externas"

"Hace más falta la rotación por el servicio de urgencia durante las guardias nocturnas y el paso de visita durante los demás turnos no solamente por la mañana con el médico egresado"

Es evidente que se considera necesario tener claridad respecto al objetivo del internado de pregrado: que los médicos en formación manifiesten y desarrollen las competencias que integran su perfil de egreso en situaciones de salud, atendiendo los programas: académico y operación.

Fase 3. Precisión de lo que se quiere mejorar o transformar

Cada uno de los equipos: 1. Administración educativa; 2 . organización y estructuración de los contenidos; 3. Formación y evaluación de las competencias; 4. Tutorías Clínicas y 5. Infraestructura y equipamiento. Al tener claro las limitaciones, precisaron que habría de mejorar tomando en cuenta los objetivos del Internado de Pregrado, la Norma Oficial Mexicana Nom. 234, los Estándares de Calidad de la AMFEM.

Para complementar la información, se realizó una entrevista de grupo focal con nueve médicos internos sobre los aspectos que necesitan mejorar en el internado de pregrado. Manifestando lo siguiente:

"... yo no sé cuáles son los objetivos"

"Se supone que es integrarnos a lo práctico, eso es lo que yo creo no, así en si no se cuáles son los objetivos de la UACH"

"Como que en realidad no tenemos este definido que es lo que somos, en cada servicio cambia, es diferente, lo que el residente quiere es lo que vas a hacer"
Ante ello propusieron que se dieran a conocer lo objetivos no sólo a los internos sino también a todo el personal, lo cual puede ser a través de un curso de inducción y valorar su cumplimiento.

Respecto a la supervisión de su práctica profesional los MIP proponen que se realice por parte de las autoridades de la Facultad:

"Yo creo que algo también muy importante sería la supervisión de que se esté llevando a cabo estos puntos no, que autoridades de la misma UACH vengan a ver como se está llevando nuestra formación como médicos internos, que se esté realmente cumpliendo los objetivos, que vengan y vean"

"En general requieren mayor supervisión ya que algunos muestran verdaderamente interés en ciertas áreas de competencia"

Consideran que es importante que la Facultad de Medicina asuma la responsabilidad del cambio e innovación educativa en el internado de pregrado.

De las rotaciones opinaron que se desarrollan de forma distinta, entre un grupo de médicos internos y otro aun cuando sea la misma área, lo cual depende según lo informan del residente:

"... muchas veces entre una rotación de un grupo de internos de rotación y el siguiente aprendemos cosas distintas incluso entre nosotros mismos en otro servicio a lo mejor nos toca con un residente que le gusta enseñar $\mathrm{y}$ terminas aprendiendo más que tu compañero que al residente que no le gusta enseñarte entonces si varía mucho el aprendizaje dependiendo de con quién te toque relacionarte"

Para mejorar el proceso de las rotaciones proponen que al "cierre" de las mismas, se explique las actividades a realizar y sus propósitos: 
"... que cada dos meses se termine y pues como se puede dar tiempo uno para una reunión y que se expliquen de nuevo, es que hay veces que recuerda unos y olvida otros y que evalúes si se llevaron a cabo los que están pasando en cada una de las rotaciones"

Este planteamiento hace referencia al programa operativo de cada Hospital, donde se precisa la organización de las actividades que realizan los MIP en cada área por las que rotan: medicina interna, pediatría, cirugía general, gineco- obstetricia, urgencias y medicina familiar, de acuerdo al programa académico de la Facultad; en el que proponen incluir aspectos éticos:

"Introducir en el currículo aspectos éticos"

"Enfatizar valores universales y ética médica"

La formación ética, de acuerdo con Aristóteles (en Ferrater, 2001) refiere a que la práctica médica se oriente a la consecución de los propósitos siendo honestos, responsables, comprometidos.... Ello implica para Tobón (2010) la reflexión continua respecto a las consecuencias de las acciones para que se orienten hacia el bienestar y se prevengan errores y situaciones no deseables.

Respecto a las tutorías clínicas, los MIP opinan que es muy necesario contar con este apoyo; siendo los residentes quienes pueden apoyarlos y ser los tutores clínicos:

“...estamos rodeadas de personas capaces que nos pueden orientar."

"y es que por ejemplo estamos nosotros más en relación con los residentes son prácticamente quienes nos enseñan o sea el conocimiento lo adquirimos de ellos si ellos no conocen los objetivos que debemos cumplir."

"Los internos deben tener más tutoría especializada para aprendizaje dirigido en cuanto a habilidades de urgencias y de procedimientos" “...el residente sería una buena opción, pero como decíamos antes que conociera los objetivos, que supiera más o menos por donde moverse para irnos guiando también a nosotros..."

Es innegable lo importante que es para los MIP contar un tutor clínico, cuya función es orientar y apoyar a los estudiantes en su proceso de formación profesional (Arias, 2000).

Fase 4. Estructuración del Modelo de gestión de calidad

Los resultados de la investigación y la problemática identificada derivó el diseño del Modelo de Gestión de Calidad que tiene como propósito: el mejoramiento continuo de los procesos formativos de los médicos internos de pregrado (MIP) por competencias, a través de una administración educativa del currículo, que plantea objetivos y metas claras (direccionar), planifica proyectos, los ejecuta y evalúa con apoyo de la Secretaría Académica, la Coordinación del Internado de Pregrado de la Facultad, los Jefes de Enseñanza de los hospitales sedes y el equipo de investigación.

Los proyectos que integran este modelo de gestión son:

1. Administración Educativa del Currículo

2. Programa Académico del Internado de Pregrado

3. Evaluación de las competencias

4. Tutorías Clínicas o Coaching y Gestión para el talento

5. Infraestructura y equipamiento. Estos proyectos constituyen los ejes centrales del modelo de gestión y al desarrollarse en un movimiento continuo, de forma interrelacionada dan respuesta a las necesidades identificadas y contribuyen a la formación integral con calidad de los médicos internos de pregrado. 
Este proceso de construcción fue asesorado presencial y virtual por el experto en GESFOC.

Dr. Sergio Tobón y Tobón.

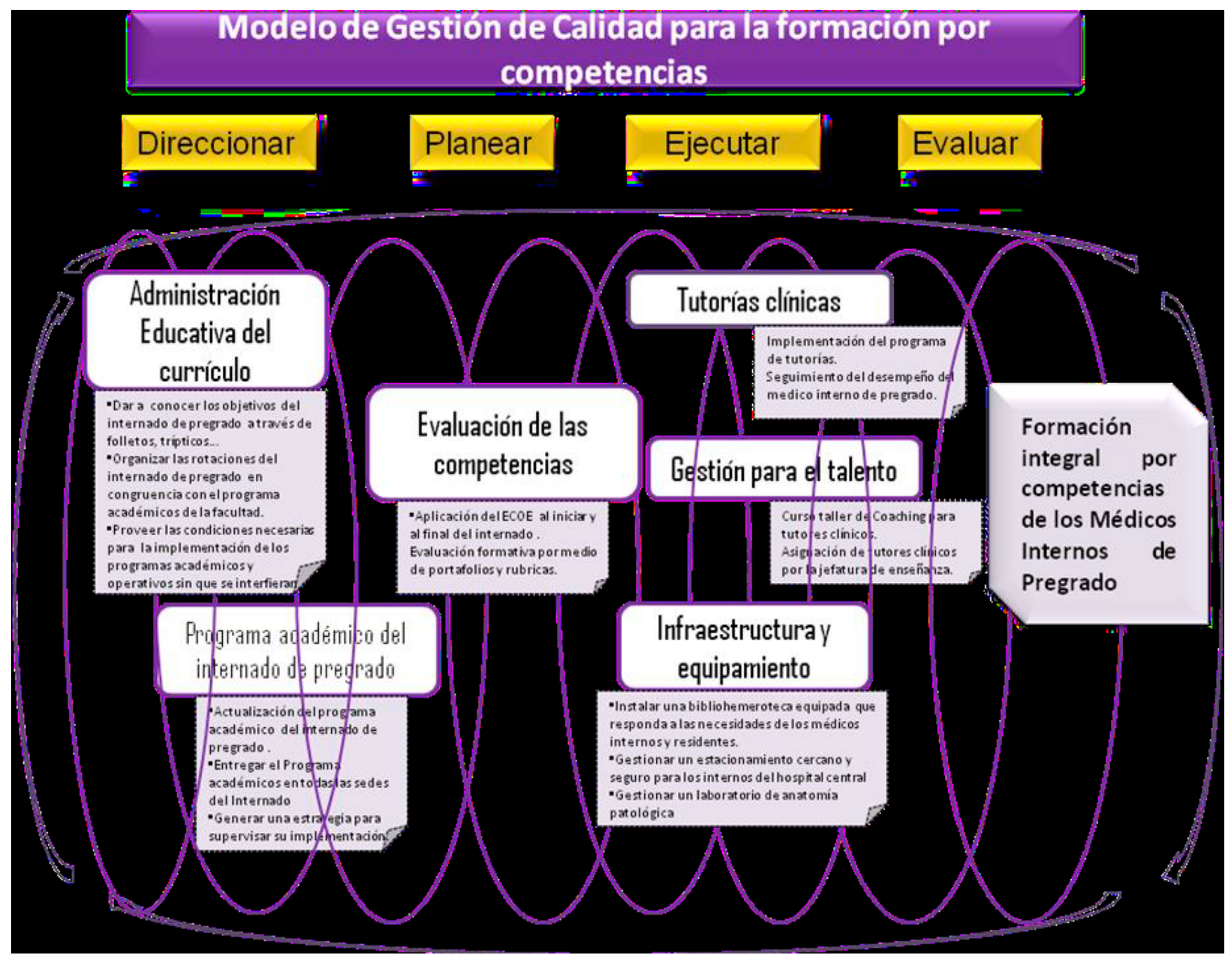

Fase V. Implementación y seguimiento

1. Se presentó el Modelo de Gestión de Calidad y el manual de procedimientos al Director y Equipo de Gobierno de la Facultad de Medicina.

2. Se revisaron cada uno de los proyectos que integran el Modelo de Gestión así como el manual de procedimientos para su implementación a través de una reunión con Secretario Académico, el Coordinador de Integración Académica y el Coordinador del Internado de Pregrado, donde se hicieron sugerencias de cambio que fueron atendidas.

3. Se reprodujeron 20 Ejemplares y 250 folletos de la guía académica del interno de pregrado y se entregaron al personal de Secretaría académica para su implementación en presencia del Director de la Facultad.

Modelo Gestión de Calidad centrado en la formación integral del Médico por Competencias. 


\section{Discusión y Conclusiones}

Los resultados muestran que el internado de pregrado es una oportunidad en la formación médica; no obstante, el desconocimiento de sus objetivos y la falta de organización de las rotaciones en congruencia con el programa académico de la Facultad, propicia que los MIP no manifiesten iniciativa e interés por su formación integral y que el personal que apoya su formación no les proporcionen asesorías que contribuyan al mejoramiento de su desempeño profesional; propiciándose que gran parte de su tiempo en el internado lo dediquen a cuestiones administrativas que limitan el desarrollo de las competencias clínicas frente a situaciones de salud.

Las limitantes observadas, reflejo de la carencias que se presentan en la administración del currículo por competencias, motivaron el diseño e implementación de un Modelo de Gestión de
Calidad para la formación de competencias (GESFOC) que plantea cuatro acciones importantes: direccionar, planear, actuar y evaluar (Tobón, 2011); lo cual responde a un ciclo de mejoramiento continuo que genera las condiciones para que el Internado de Pregrado propicie el fortalecimiento de las competencias profesionales de los MIP frente a pacientes en las diferentes rotaciones, con apoyo y asesoría del tutor clínico y del docente. Interesa egresar médicos integralmente desarrollados, que contribuyan a la prevención y den respuesta a los problemas de salud con idoneidad $y$ compromiso ético a través de cinco proyectos: administración educativa del currículo, infraestructura y equipamiento, organización y estructura de contenidos, formación por competencias y tutorías clínicas, generados de forma participativa, que se implementan con soporte en un manual de procedimientos. 


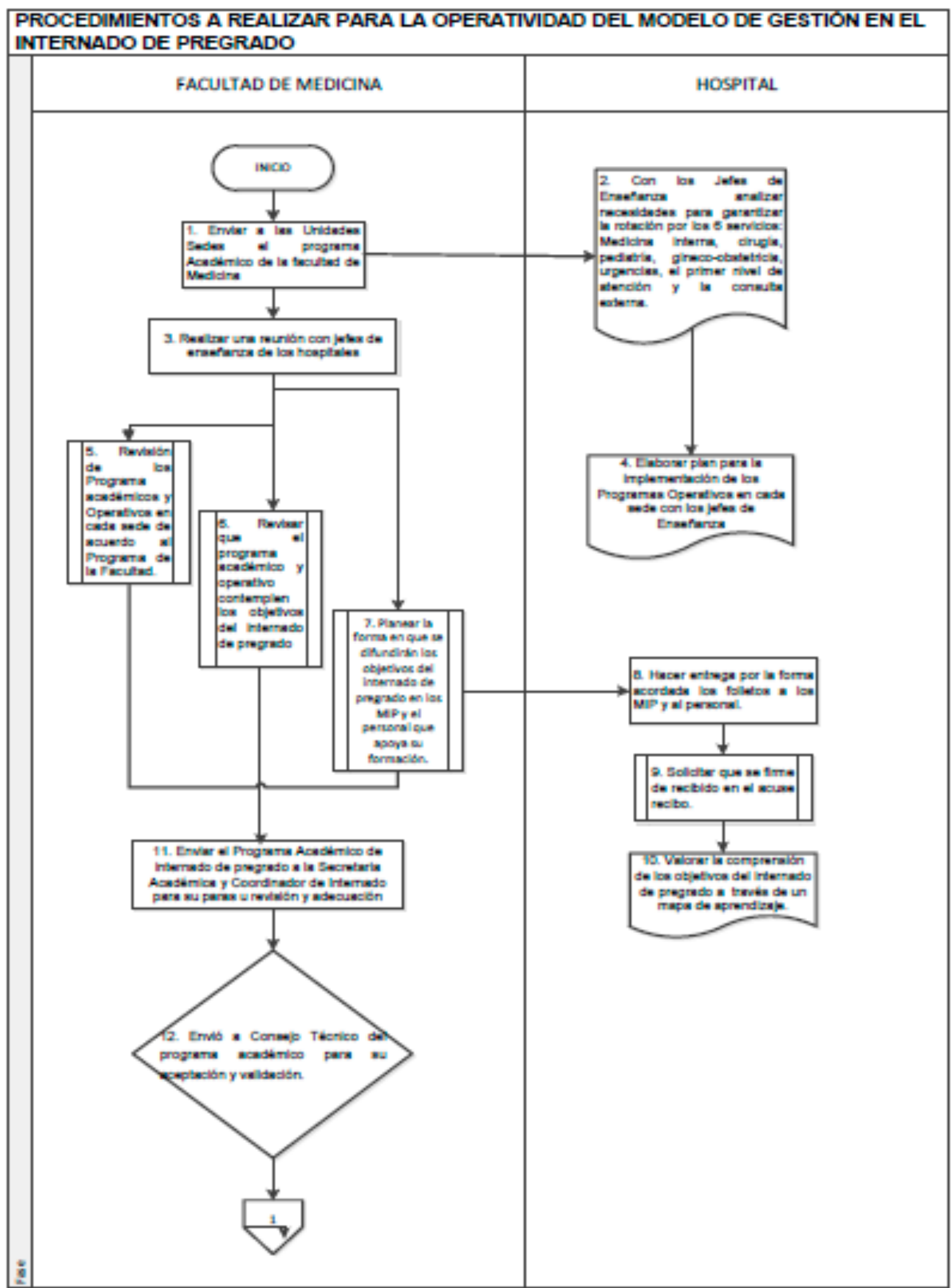

Modelo Gestión de Calidad centrado en la formación integral del Médico por Competencias. 
RECIE. Revista Electrónica Científica de Investigación Educativa

Vol. 1, núm. 2, enero-diciembre 2013, pp. 339-350.

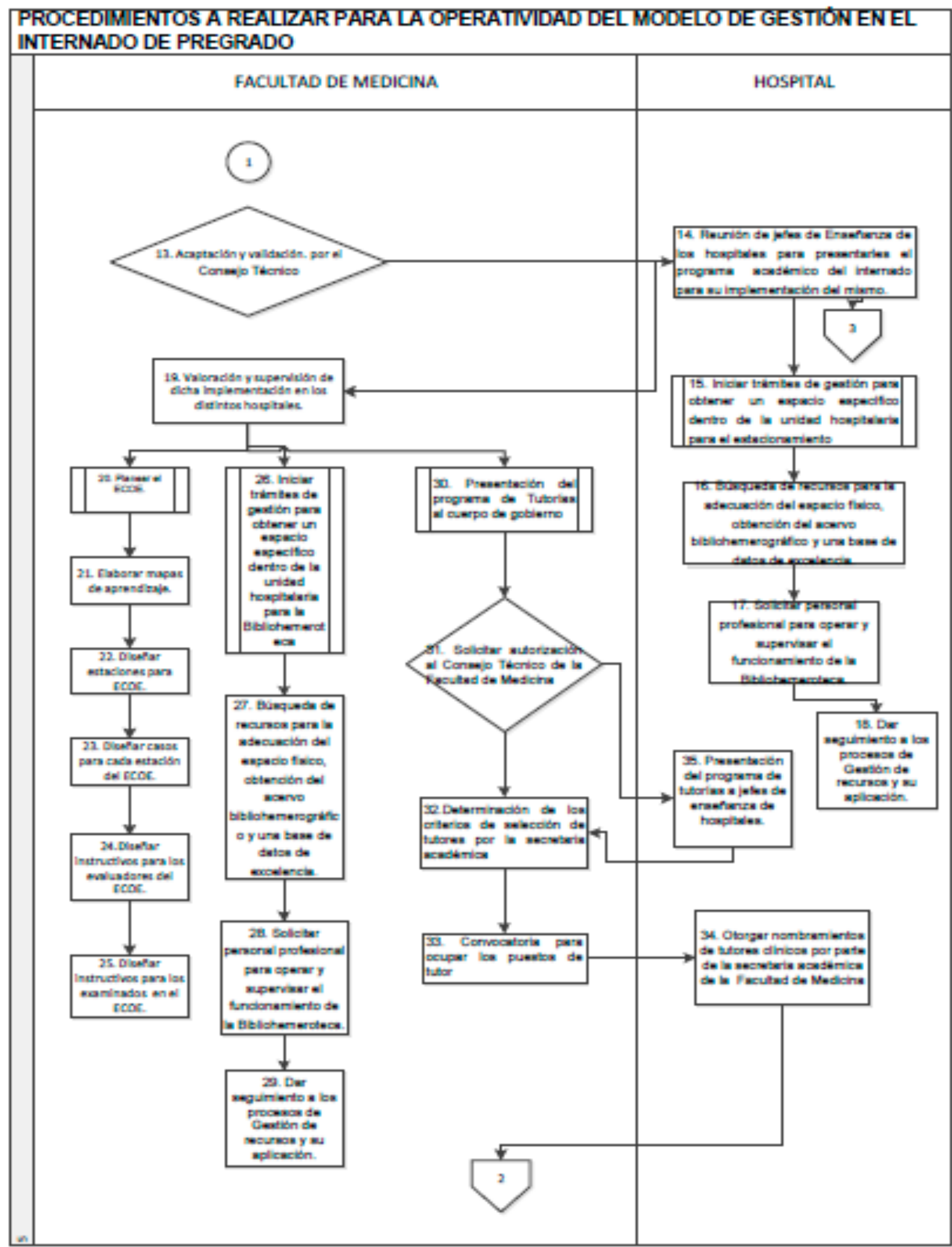

Parra Acosta, H.; López González, J. C.; y Martínez Tapia, M. 


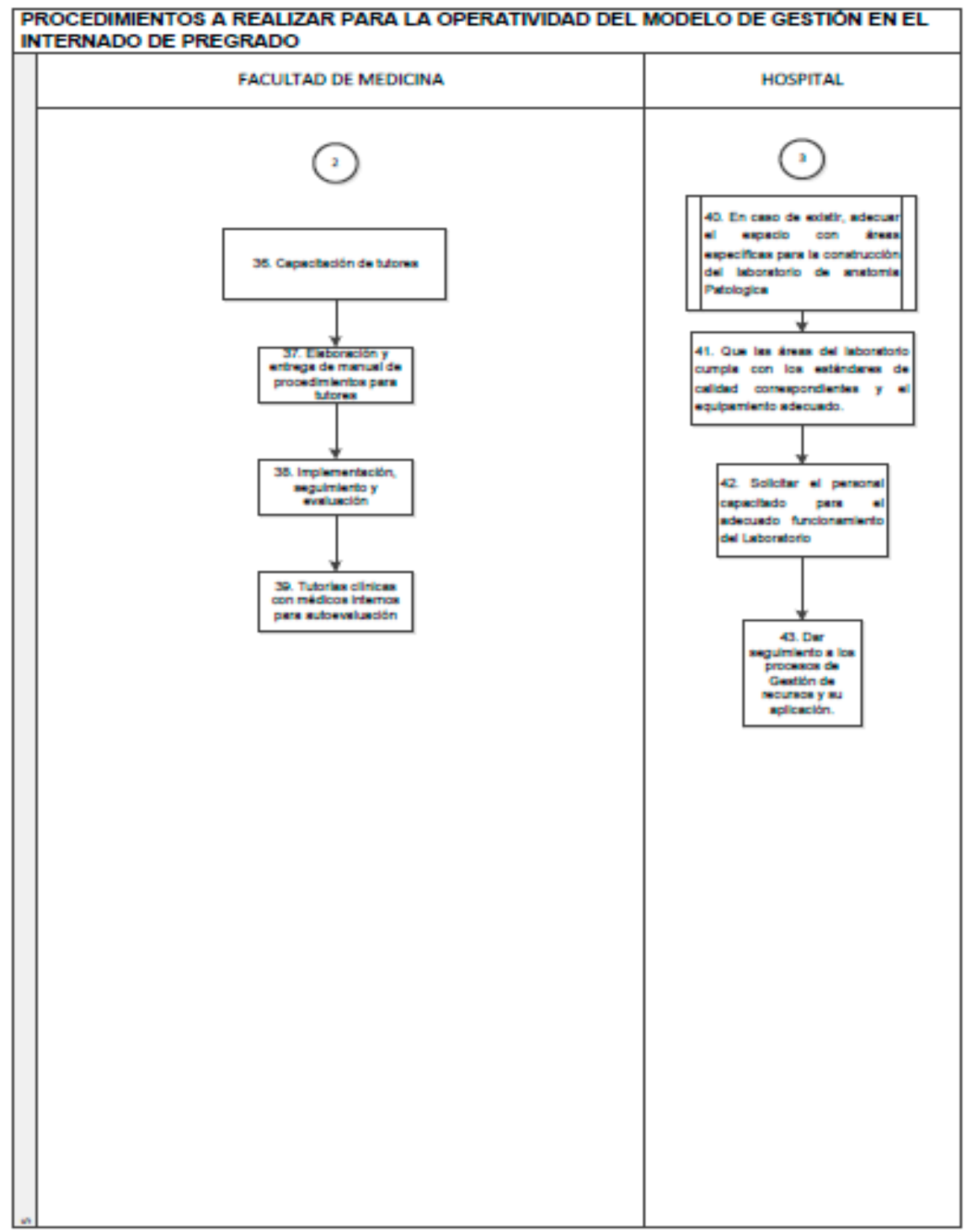

Modelo Gestión de Calidad centrado en la formación integral del Médico por Competencias. 


\section{Referencias}

Barabatarlo y Z, A. (1995) InvestigaciónAcción: Una didáctica para la formación de profesores. Universidad Autónoma Nacional. México D.F.

CIFRHS (1983) Documento: Criterios e Indicadores para estructuración de guías para evaluación de programas en carreras de la salud. XXVII Reunión.

Duncan, W. J. (2000). Las ideas y la práctica de la administración: los principales desafíos en la era moderna (M. d. P. Carril, Trad.). México, D.F.: Oxford.

Ferrater, M. J. (2001) Diccionario de Filosofía. ED. Ariel Filosofía.
FMUACH, (2010) Evaluación Integral al Proceso de Reforma Curricular y Académica.

Guzmán (2009) Fases y operaciones metodológicas en la investigación educativa. Durango, Durango México.

Norma Oficial Mexicana Nom-234-SSA1-2003, Utilización de campos clínicos Para ciclos clínicos e internado de pregrado.

Parra, A. H. et al. (2011) La administración educativa del Currículo y sus implicaciones en el desempeño profesional de los médicos internos. Facultad de Medicina de la UACH.

Tobón T, S (2010) Formación integral y competencias: Pensamiento complejo, currículo, didáctica y evaluación. 3ra. Ed. Bogotá ECOE Ediciones. 